\title{
AUTOCONHECIMENTO: CONTRIBUIÇÕES DA PESQUISA BÁSICA ${ }^{1}$
}

\author{
Alessandra da Silva Souza* \\ Josele Abreu-Rodrigues ${ }^{\#}$
}

\begin{abstract}
RESUMO. Skinner propõe ser o autoconhecimento gerado por contingências sociais que colocam os relatos do indivíduo sob o controle do seu próprio comportamento. Esse argumento tem sido sustentado por uma série de investigações, tanto com sujeitos humanos quanto com sujeitos não humanos, as quais têm indicado que diferentes aspectos das relações entre o comportamento de um organismo e suas conseqüências podem exercer controle discriminativo sobre as descrições que esse organismo faz de tais relações (auto-relato). O objetivo do presente trabalho é apresentar uma revisão da literatura sobre discriminação de contingência, com ênfase nas funções controladoras de certas características das contingências que mantêm o comportamento a ser descrito e daquelas que mantêm o comportamento de auto-relatar. Adicionalmente, algumas implicações desses estudos para a vida cotidiana são discutidas.
\end{abstract}

Palavras-chave: autoconhecimento, discriminação de contingência, auto-relato.

\section{SELF-KNOWLEDGE: SOME CONTRIBUTIONS FROM BASIC RESEARCH}

\begin{abstract}
Skinner proposed that self-knowledge is a consequence of social contingencies which place the individuals' reports under the control of their own behavior. The above has been corroborated by several investigations on human and nonhuman subjects. Investigations have shown that different aspects of the relationships between the organism's behavior and its consequences may have a discriminative control on the organism's descriptions (self reports). A review of the literature on contingency discrimination is given, highlighting the controlling functions of certain characteristics of contingencies that maintain the behavior to be described and those that maintain the self-report behavior. Several implications of these studies on day-to-day life are also discussed.
\end{abstract}

Key words: Self-knowledge, contingency discrimination, self-report.

\section{AUTO CONOCIMIENTO: CONTRIBUCIONES DE LA INVESTIGACIÓN BÁSICA}

RESUMEN. Skinner propone ser el auto conocimiento generado por contingencias sociales que colocan los relatos del individuo bajo el control de su propio comportamiento. Ese argumento ha sido sostenido por una serie de investigaciones, tanto con sujetos humanos, como con sujetos no humanos, las cuales han indicado que distintos aspectos de las relaciones entre el comportamiento de un organismo y sus consecuencias poden ejercer control discriminativo sobre las descripciones que ese organismo hace de tales relaciones (auto-relato). El objetivo del presente trabajo es presentar una revisión de la literatura sobre discriminación de contingencia, con énfasis en las funciones controladoras de ciertas características de las contingencias que mantienen el comportamiento a ser descrito y de aquéllas que mantienen el comportamiento de auto-relatar. Adicionalmente, son discutidas algunas implicaciones de esos estudios para la vida cotidiana.

Palabras-clave: auto conocimiento, discriminación de contingencia, auto-relato.

Skinner (1974/2004) discutiu a questão sobre o que uma pessoa pode conhecer a respeito do seu próprio comportamento. Essa questão, em sua opinião, só poderia ser respondida investigando-se as "contingências que produzem tanto um eu cognoscente quanto um eu conhecido" (p.146). Para Skinner (1974/2004), o autoconhecimento é um produto social, sendo gerado por contingências especiais estabelecidas pela comunidade verbal na qual o indivíduo está inserido. Essas contingências

\footnotetext{
Apoio: CAPES.

* Mestre em Psicologia. Doutoranda do Instituto de Psicologia da Universidade de Brasília.

\# Professora do Instituto de Psicologia da Universidade de Brasília, PhD. em Psicologia, em Análise do Comportamento, West Virginia University, USA.
} 
colocam o comportamento verbal do indivíduo (autorelato) sob o controle do seu próprio comportamento não verbal, tornando-o um "eu cognoscente". É a identificação das contingências que estabelecem tanto o comportamento não-verbal quanto a descrição desse comportamento (auto-relato) que torna possível a compreensão da origem e manutenção do autoconhecimento. A coerência, a mentira, a omissão, a negação, por exemplo, são fenômenos comportamentais que podem ser compreendidos a partir da avaliação das contingências de reforçamento e punição que mantêm o autoconhecimento (Beckert, 2005).

A relevância aplicada desse tema é inestimável. Nas diversas relações sociais (e.g., familiares, profissionais, educacionais) é freqüente o indivíduo ser solicitado a relatar o seu próprio comportamento ou as contingências a que foi exposto, uma vez que os autorelatos permitem que a comunidade entre em contato com eventos aos quais apenas o indivíduo tem acesso (e.g., sentimentos, desejos, pensamentos, idéias). Além disso, esses auto-relatos fornecem dicas importantes para a comunidade sobre as variáveis controladoras do comportamento do indivíduo (isto é, o "eu conhecido"). Adicionalmente, esse conhecimento é importante para o próprio indivíduo, já que a discriminação das variáveis das quais o seu próprio comportamento é função pode favorecer o estabelecimento de comportamentos mais efetivos.

Conforme assinalado por Skinner (1953/2000), o comportamento verbal é regido pelos mesmos princípios do comportamento não verbal. Com base nesse preceito, Shimp (1983) argumenta que estudos sobre o auto-relato podem ser realizados não somente com sujeitos humanos, mas também com sujeitos não humanos, por meio da utilização de procedimentos experimentais que permitam acessar o controle discriminativo que um comportamento exerce sobre outro comportamento do próprio organismo. Ou seja, um animal não humano pode 'relatar' seu comportamento ou, alternativamente, as contingências de reforçamento às quais foi exposto. Esse argumento tem sido confirmado por diversos pesquisadores (Commons, 1979; Hobson, 1975; Killeen, 1978; Kramer, 1982; Lattal, 1975, 1979; Lionello-DeNolf \& Urcuioli, 2003; Maki, 1979; Reynolds, 1966; Reynolds \& Catania, 1962; Rilling \& McDiarmid, 1965; Shimp, 1981, 1982, 1983; Ziriax \& Silberberg, 1978).

Segundo Skinner (1953/2000), autoconhecimento compreende diversos comportamentos, tais como, discriminar que 'fez alguma coisa', que 'está fazendo alguma coisa', que 'tende a fazer alguma coisa' e que 'determinadas variáveis são responsáveis por seu comportamento'. Esses comportamentos discriminativos, ou mais precisamente, as contingências que geram e mantêm esses comportamentos, têm sido investigados não somente sob o rótulo de autoconhecimento, mas também sob os rótulos de autotato, auto-relato, autoconsciência, correspondência não verbal-verbal (ou fazer-dizer), discriminação de contingência, dentre outros (Dymond \& Barnes, 1997). No presente trabalho, serão discutidos apenas os estudos de discriminação de contingência, os quais definem o autoconhecimento como o comportamento de descrever o próprio comportamento. Mais especificamente, serão revisados aspectos das relações de contingência que podem assumir funções discriminativas sobre o autorelato, tais como as características dos estímulos e do próprio responder. Serão também destacadas características das contingências que mantêm o autorelato. Os estudos revisados abrangem tanto a literatura referente a organismos humanos quanto a referente a não humanos.

\section{DISCRIMINAÇÃO DE CONTINGÊNCIA}

As investigações sobre as propriedades discriminativas das contingências utilizam prioritariamente procedimentos de discriminação condicional. $\mathrm{O}$ procedimento experimental padrão, denominado de escolha de acordo com o modelo, será detalhado a seguir de forma a facilitar a compreensão dos estudos realizados nessa área, os quais serão posteriormente discutidos.

\section{Escolha de Acordo com o Modelo}

O procedimento de escolha de acordo com o modelo compreende cinco etapas: a apresentação de um estímulo-modelo (estímulo condicional - $\mathrm{S}^{\mathrm{C}}$ ), um intervalo, apresentação de estímulos de comparação (estímulos discriminativos - $S^{D}$ e $S^{\Delta}$ ), emissão da resposta de escolha e apresentação da consequiência. $\mathrm{O}$ modelo estabelece qual estímulo funcionará como $\mathrm{S}^{\mathrm{D}}$ e $\mathrm{S}^{\Delta}$ em cada situação, sendo que a escolha do estímulo de comparação selecionado pelo experimentador como correspondente ao modelo $\left(\mathrm{S}^{\mathrm{D}}\right)$ é seguido pela apresentação do reforço $\left(S^{R}\right)$ e a escolha do outro estímulo de comparação $\left(\mathrm{S}^{\Delta}\right)$ é seguida por um período de suspensão total (blackout - BO) ou parcial (timeout - TO) da contingência reforçadora.

Nas investigações sobre discriminação de contingências, esse procedimento apresenta as 
seguintes características: (1) a relação respostaconseqüência é utilizada como modelo; (2) luzes coloridas ou a posição das luzes são utilizadas como comparações; e (3) a relação entre esses estímulos é construída experimentalmente. $\mathrm{O}$ elo do modelo, portanto, refere-se ao comportamento (ou contingência) na presença exclusiva do modelo e o elo de auto-relato consiste no comportamento de escolha entre as comparações.

Esse procedimento de escolha tem sido empregado nas pesquisas sobre as propriedades discriminativas das contingências de reforçamento, uma vez que, conforme salientado por Lattal (1975), essa metodologia fornece um índice da função discriminativa da contingência independentemente dos comportamentos diretamente controlados por esta mesma contingência. Dessa forma, os estímulos $\left(S^{\mathrm{D}} \mathrm{e}\right.$ $\left.S^{R}\right)$ presentes durante o elo do modelo e aqueles $\left(S^{D}\right.$ e $S^{R}$ ) durante o elo do auto-relato são diferentes, bem como as respostas por eles controladas. Ou seja, o comportamento que tem sua freqüência alterada em função da contingência em vigor durante o modelo é diferente do comportamento que é controlado por essa contingência enquanto estímulo discriminativo. Essa característica permite que as funções reforçadoras e discriminativas da relação resposta-conseqüência possam ser isoladas e o grau de correlação entre essas funções possa ser avaliado.

Nos tópicos seguintes serão descritos estudos que demonstraram o controle discriminativo de diferentes relações de contingência sobre o auto-relato. Duas variáveis de controle serão salientadas: as características do comportamento selecionado pelas contingências e as características dos estímulos presentes nessas contingências.

\section{Propriedades discriminativas do responder}

Número de respostas. Rilling e McDiarmid (1965) realizaram um dos primeiros estudos sobre as propriedades discriminativas das contingências de reforçamento utilizando pombos como sujeitos experimentais. Esses autores investigaram se o número de respostas emitidas exerceria controle discriminativo sobre o auto-relato. $\mathrm{O}$ modelo consistia em um esquema misto razão fixa (FR) razão fixa (FR). No início da tentativa o disco central era iluminado e o computador selecionava uma razão de respostas. Após completar a razão previamente selecionada, duas alternativas de auto-relato eram apresentadas, ou seja, dois discos laterais eram iluminados. $\mathrm{O}$ treino envolveu, em um primeiro momento, a discriminação de valores bastante distintos do esquema FR, tais como
5 e 50. Em seguida, a diferença entre os dois esquemas foi gradualmente reduzida, aumentando-se a razão do esquema FR de menor valor. Os resultados encontrados apontaram que a diferença entre os valores absolutos das razões afetou as escolhas, sendo que à medida que esta diferença tendia a zero, a acurácia do auto-relato (isto é, a porcentagem de escolhas corretas) tendeu ao acaso (ver também Hobson, 1975; Pliskoff \& Goldiamond, 1966; Shimp, 1982).

Duração da resposta. Ziriax e Silberberg (1978) investigaram se a duração da pressão de um disco poderia assumir funções discriminativas sobre o autorelato utilizando quatro pombos como sujeitos experimentais. No início da tentativa, um disco central era iluminado por uma luz azul e o computador selecionava randomicamente um dentre três modelos: (a) resposta de duração longa (pressões com duração entre 60 e $180 \mathrm{~ms}$ ); (b) respostas de duração curta (pressões com duração entre 0 e $20 \mathrm{~ms}$ ); e (c) sem resposta. Após a emissão do modelo selecionado, três discos eram iluminados randomicamente com as cores vermelho, branco e verde, e serviam como alternativas de auto-relato. Os resultados indicaram que a acurácia do auto-relato dos três tipos de tentativa foi aproximadamente $60 \%$ para a maioria dos pombos. Em seguida, os estímulos discriminativos foram invertidos para todos os modelos Com essa inversão, a acurácia do auto-relato diminuiu para níveis próximos do acaso (33\%), mas, ao longo das sessões, a acurácia gradualmente assumiu valores similares àqueles observados antes da reversão. Esses resultados indicam que a relação entre duração da resposta (isto é, um estímulo proprioceptivo) e sua conseqüência pode exercer controle discriminativo sobre o auto-relato.

Intervalo entre Respostas (IRT). Shimp (1981) investigou as propriedades discriminativas da contingência utilizando diferentes IRTs como modelo para o auto-relato de pombos (ver também Reynolds, 1966; Shimp, 1983). No elo do modelo, um disco central era iluminado por uma luz branca e, após um intervalo variável (VI) de 20 s, o primeiro IRT que atendesse ao critério previamente selecionado pelo computador produzia a mudança para o elo do autorelato, isto é, a iluminação de duas chaves laterais pelas luzes vermelha e verde. A cada tentativa o computador selecionava randomicamente um dentre dois IRTs (um longo e um curto). O valor do IRT longo foi mantido constante, enquanto o valor do IRT curto foi gradualmente aumentado ao longo das sessões. Os resultados mostraram que a acurácia do auto-relato diminuiu com o aumento da similaridade entre os IRTs, sugerindo que o grau de controle dos 
estímulos presentes na contingência varia de forma inversamente proporcional à similaridade entre os modelos. Essa conclusão é similar àquela apresentada por Hobson (1975) e Rilling e McDiarmid (1965) com relação à manipulação do número de respostas.

Taxa de respostas. Okouchi e Songmi (2004) avaliaram as propriedades discriminativas da taxa de respostas com participantes humanos. Durante o elo do modelo estava em vigor um esquema misto com dois componentes, FR e reforçamento diferencial de taxas baixas (DRL), os quais geram taxas de respostas altas e baixas, respectivamente. Após completar o esquema, os participantes relatavam seu desempenho na contingência selecionando uma dentre duas alternativas, isto é, "Rápido" ou "Devagar". Os participantes foram expostos a duas condições: uma onde havia reforçamento diferencial (RD) para a ausência de correspondência fazer-dizer e uma para a qual não havia reforçamento diferencial (NOT-RD) dos auto-relatos. Em tentativas de sondagem, um esquema VI foi apresentado como modelo, após o qual nenhum auto-relato era reforçado. Essas tentativas objetivavam verificar se o controle discriminativo havia sido estabelecido pelos esquemas FR e DRL ou pela taxa de respostas produzidas por esses esquemas: caso o auto-relato fosse uma função dos esquemas, não deveria haver relação entre a taxa emitida no esquema VI e o auto-relato. Os resultados indicaram que, na condição RD, os participantes relataram "Rápido" para o esquema DRL e "Devagar" para o esquema FR, mostrando ausência de correspondência. Na condição NOT-RD, os participantes tenderam a relatar acuradamente o modelo. Nas tentativas de sondagem, os resultados mostraram que o auto-relato foi uma função da taxa de respostas emitida: taxas altas foram seguidas pelo auto-relato "Devagar" e taxas baixas foram seguidas pelo auto-relato "Rápido" na condição RD, enquanto o inverso ocorreu na condição NOT-RD. Esse resultado indicou que a taxa de respostas assumiu propriedades discriminativas sobre o auto-relato, dependendo da história prévia. Além disso, esse resultado é comparável àqueles obtidos com animais infra-humanos, os quais indicaram que taxas de respostas diferentes podem assumir funções de $\mathrm{S}^{\mathrm{D}}$ (Lionello-DeNolf \& Urcuioli, 2003; Urcuioli, 1985; Urcuioli \& DeMarse, 1994).

Presença versus ausência do responder. Lattal (1975) investigou o controle discriminativo exercido pela ocorrência ou não da resposta, utilizando como modelo um esquema misto DRL e reforçamento diferencial de outros comportamentos (DRO). No elo do modelo, o disco central era iluminado por uma cor branca e o computador selecionava qual esquema estaria em vigor a cada tentativa. Após completar o requerimento do modelo, o disco central era apagado e dois discos laterais eram iluminados pelas cores vermelho e verde que serviam como alternativas de auto-relato. Após essa fase de treino, os estímulos discriminativos correlacionados com cada esquema foram invertidos em duas condições subseqüentes, de forma a acessar o controle dos estímulos. A acurácia do auto-relato se manteve superior a $75 \%$ na fase de treino, indicando que as contingências de reforçamento assumiram funções discriminativas para as respostas de escolha. As duas inversões dos estímulos discriminativos foram seguidas por um declínio acentuado na acurácia do auto-relato e uma posterior recuperação gradual da mesma ao longo das sessões. Mais uma vez essas evidências sugerem que pombos podem relatar acuradamente a relação respostaconseqüência, identificando corretamente as contingências que exigem que um comportamento-alvo seja emitido (DRL) daquelas contingências que exigem a não-emissão desse comportamento (DRO).

Complexidade do responder. Contingências que selecionam operantes complexos, como a variação e a repetição comportamental, também podem assumir funções discriminativas sobre o auto-relato. No estudo de Souza (2006), pombos foram expostos a uma situação de escolha de acordo com o modelo na qual, durante o elo do modelo, estava em vigor um esquema misto Variação/Repetição. A tarefa consistia em formar seqüências de quatro respostas distribuídas em dois discos iluminados pela luz vermelha. $\mathrm{Na}$ contingência de Variação estava em vigor um esquema percentil, no qual sequiências com frequiência relativa igual ou inferior a $10 \%$ eram reforçadas. $\mathrm{Na}$ contingência de Repetição, a emissão de apenas duas seqüências específicas (dentre as 16 possíveis) produzia reforços. Após a exposição ao modelo pelo tempo mínimo de $01 \mathrm{~min}$, o primeiro reforço era seguido pela apresentação do elo do auto-relato. $\mathrm{O}$ auto-relato correto dependia da contingência que estava em vigor anteriormente no modelo: se fosse variação, o animal deveria escolher o disco iluminado por uma luz branca; se fosse repetição, o disco iluminado por uma luz verde deveria ser escolhido. Após o estabelecimento de auto-relatos estáveis e acurados (75\% de escolhas corretas durante cinco sessões consecutivas) na fase de linha de base, o critério de variação (percentil) foi sistematicamente manipulado em uma fase de teste. Os valores do percentil foram apresentados na seguinte ordem: $15 \%, 25 \%, 50 \%$, $75 \%, 100 \%, 50 \%, 25 \%, 15 \%$ e $10 \%$.

Durante a linha de base, as contingências de Variação e Repetição assumiram funções 
discriminativas sobre o auto-relato para três dos quatro pombos. Na fase de teste, a acurácia do auto-relato de Variação foi uma função inversa do percentil. Uma vez que quanto maior (menor) o percentil, menor (maior) o nível de variabilidade exigido, o comportamento apresentado no elo do modelo foi progressivamente menos (mais) variável e, conseqüentemente, a porcentagem de auto-relatos de variação diminuiu (aumentou). Esse resultado sugere que auto-relatos acurados podem ser obtidos mesmo quando os comportamentos relatados estão sob o controle de contingências complexas.

Sumarizando, esses dados mostram que o comportamento pode assumir funções discriminativas sobre o auto-relato quando todas as demais condições são mantidas constantes, isto é, taxa de reforços, magnitude do reforço, frequiência e duração do modelo. Contudo, outros componentes da contingência também podem assumir funções discriminativas, como, por exemplo, a freqüência do modelo, a densidade de reforços e o intervalo entre reforços.

\section{Propriedades discriminativas dos estímulos}

Freqüiência do modelo. Lattal (1979) procurou verificar se a freqüência de ocorrência do modelo poderia enviesar o auto-relato. Para tanto, um esquema misto DRL-DRO foi utilizado como modelo, sendo manipulada a proporção entre as ocorrências do componente DRL e as ocorrências do componente DRO. As proporções utilizadas foram apresentadas na seguinte ordem: 0,$5 ; 0,75 ; 0,25 ; 0,0 ; 1,0 ; 0,07 ; 0,93$; e 0,5 . Após cada modelo, duas alternativas de auto-relato eram apresentadas. Os resultados indicaram que quanto maior a proporção de DRL, maior a tendência a relatar DRL, independentemente de o esquema anteriormente apresentado no modelo ser DRL ou DRO. Dessa forma, com o aumento no número de modelos DRL, os pombos tenderam a aumentar suas chances de acerto respondendo predominantemente na chave correlacionada com o esquema DRL no elo de autorelato, embora o predomínio de relatos DRL tenha implicado também no aumento de respostas incorretas. Esse resultado sugere que o aumento na frequiência do evento a ser detectado (modelo) produz vieses no relato desse evento (ver também Jones \& Davison, 1998, Parte 2).

Intervalo entre modelo e comparação. Um dos objetivos do estudo de Lattal (1975) foi avaliar se o intervalo de tempo entre o elo do modelo e o elo do auto-relato afetaria a função discriminativa exercida pela presença $v s$. ausência do responder sobre o autorelato de pombos. Para tanto, no elo do modelo estava em vigor um esquema misto DRL-DRO. Após completar o requerimento no modelo, duas alternativas de auto-relato eram apresentadas. Após o estabelecimento de controle discriminativo (acurácia superior a $80 \%$ ) foi introduzido um intervalo entre o modelo e o elo do auto-relato que foi gradualmente aumentado ao longo das sessões. $\mathrm{O}$ aumento do intervalo produziu diminuições proporcionais na acurácia do auto-relato, até que com intervalos longos o controle discriminativo foi eliminado. Esse resultado sugere, portanto, que a passagem do tempo é uma variável que afeta a propriedade discriminativa das contingências (ver também Kramer, 1982, Experimentos 1 e 2).

Essa relação inversa entre duração do intervalo e acurácia do auto-relato também foi observada quando o modelo compreendia IRTs diferentes (Shimp, 1981), estímulos coloridos (Lattal \& Doepke, 2001) e razão de respostas (Shimp, 1982).

Densidade de reforços. Commons (1979) investigou a discriminabilidade da densidade de reforços, ou seja, do número de reforços por unidade de tempo, utilizando pombos como sujeitos. Para tanto, no elo do modelo, um dentre dois esquemas era apresentado no disco central: um esquema de alta densidade de reforços ou um esquema de baixa densidade de reforços. O modelo ficava em vigor por $12 \mathrm{~s}$, sendo que, a cada $3 \mathrm{~s}$, a primeira resposta emitida era elegível para o reforçamento segundo a probabilidade de reforço determinada pelo computador naquela tentativa $\left[p\left(\mathrm{~S}^{\mathrm{R}}\right)=0,25\right.$ ou $\left.p\left(\mathrm{~S}^{\mathrm{R}}\right)=0,75\right]$. Dessa forma, no esquema de alta densidade eram liberados três reforços, em média, a cada modelo, enquanto no esquema de baixa densidade era liberado um reforço, em média, a cada apresentação do modelo. Após o término do elo do modelo, a luz central era apagada e dois discos laterais, iluminados pelas cores vermelha e verde, serviam como alternativas de auto-relato. Três dos quatro pombos apresentaram auto-relatos acurados (acima de $80 \%$ ), indicando que a densidade de reforços pode exercer controle discriminativo sobre o auto-relato.

Intervalo entre reforços (IRI). Okouchi (2003) realizou um estudo que teve por objetivo investigar as propriedades discriminativas da distribuição temporal dos reforços. Para tanto, estudantes universitários foram expostos a um esquema misto FR-DRL cujos valores foram manipulados de forma a produzir IRIs curtos ou longos (FR com IRI curto e DRL com IRI longo ou vice-versa) na fase de treino. No Experimento 1, a fase de treino foi seguida por uma mudança não sinalizada para a fase de teste que compreendeu um esquema misto FI FI cujos IRIs eram 
semelhantes aos IRIs (curtos ou longos) produzidos pelo esquema misto FR-DRL. Os resultados mostraram que a taxa de respostas em cada componente do esquema FI foi uma função do treino prévio. Se o IRI do esquema FI era longo e no treino o esquema FR estava correlacionado com o IRI longo, a taxa de respostas na fase de teste foi alta; porém, se o esquema DRL estava correlacionado com o IRI longo, a taxa de respostas foi baixa. Resultados semelhantes foram obtidos com o IRI curto. No Experimento 2, foi testada a generalização da propriedade discriminativa dos IRIs longo e curto. Para avaliar a generalização, tentativas de sondagem foram introduzidas após a fase de treino, durante as quais um esquema misto FI-FI com valores que variavam de 5 a $40 \mathrm{~s}$ foi implementado. Nessas tentativas, à medida que o valor do IRI aumentava, a taxa de respostas diminuía para o grupo que havia sido exposto a uma história de IRI longo com taxa baixa de respostas; entretanto, para o grupo com uma história de IRI longo e taxa alta de respostas, foram observados aumentos correspondentes na taxa de respostas. Esses resultados sugerem que a distribuição temporal dos reforços pode assumir funções discriminativas. Todavia, esse estudo não utilizou um procedimento de escolha de acordo com o modelo, não permitindo, portanto, investigar diretamente o controle discriminativo dessa propriedade sobre um outro comportamento, isto é, o auto-relato.

Os estudos apresentados anteriormente indicaram que diferentes propriedades da relação respostaconsequiência podem assumir funções discriminativas. Alguns autores buscaram também investigar as propriedades controladoras de determinadas características das contingências que mantêm o próprio auto-relato.

\section{Variáveis que afetam o auto-relato}

Manipulações em determinadas características da contingência que estabelece e mantém o auto-relato afetam sistematicamente a correspondência entre esse comportamento e aquele previamente emitido no modelo, conforme indicado nos estudos abaixo discutidos.

Magnitude do reforço. Killeen (1978) avaliou os efeitos da magnitude do reforço sobre a acurácia do auto-relato. No elo do modelo, um disco central era iluminado. Respostas no disco produziam a mudança para o elo do auto-relato com uma probabilidade de 0,05 . Adicionalmente, o computador produzia pseudorespostas com a mesma taxa apresentada pelo sujeito e com a mesma probabilidade de produzir a mudança para o elo do auto-relato. A tarefa consistia em discernir se a mudança para o elo do auto-relato havia sido produzida pela resposta do pombo (controle da mudança) ou pelo computador. Após o estabelecimento dessa discriminação, diferentes magnitudes de reforço foram fornecidas para os auto-relatos de controle. Aumentos na magnitude do reforço geraram acréscimos na porcentagem de vezes em que os animais relatavam controle da mudança, ou seja, produziram um viés para a escolha dessa alternativa (ver também Commons, 1979).

Uma vez que aumentos na magnitude produzem vieses no auto-relato, é possível que aumentos na probabilidade do reforço para o auto-relato de uma alternativa produzam resultados comparáveis. Essa possibilidade foi investigada no estudo descrito a seguir.

Probabilidade de reforço. Jones e Davison (1998, Parte 1) investigaram se aumentos na probabilidade do reforço para o auto-relato de uma alternativa afetariam a correspondência entre o autorelato e o modelo. Para tanto, no elo do modelo estava em vigor um esquema concorrente VI-VI. Cada componente era sinalizado por uma intensidade (alta ou baixa) de uma luz amarela. Após cada reforço liberado no esquema concorrente, duas alternativas de autorelato (discos iluminados pelas cores vermelha e verde) eram apresentadas e o pombo deveria relatar em qual componente do esquema concorrente (luz alta ou luz baixa) o último reforço havia sido liberado. Respostas no disco correto eram seguidas por reforço com uma probabilidade $x$, que foi manipulada ao longo das condições, e respostas no disco incorreto eram seguidas por um BO. As probabilidades do reforço para o autorelato da alternativa vermelha foram: 0,$5 ; 0,9 ; 0,2 ; 0,8$; 0,1 ; e 0,5 . As probabilidades de reforço para o autorelato da alternativa verde em cada condição foram o complemento da probabilidade utilizada no modelo vermelho. Manipulações na probabilidade do reforço não afetaram a distribuição de respostas no esquema concorrente em vigor no elo do modelo, mas produziram vieses para 0 auto-relato da alternativa vermelha, de modo que quanto maior a probabilidade de reforço, maior o viés observado. Esses resultados são comparáveis àqueles obtidos com 0 aumento na magnitude do reforço.

Punição do auto-relato. No estudo de Critchfield (1993), estudantes universitários tinham que executar uma tarefa, durante o elo do modelo, que produzia ou não ganho de pontos; em seguida, durante o elo do auto-relato, deveriam indicar se haviam ou não acertado a tarefa. Os resultados indicaram que os estudantes apresentaram um viés para o relato de "acerto". Esse autor sugeriu, então, que uma possível 
história de reforçamento contingente à emissão de relatos de sucesso e de punição contingente a relatos de insucesso poderia ter produzido esse viés.

Essa sugestão foi investigada por Sanabio e Abreu-Rodrigues (2002, Experimento 1), em um estudo em que a história de punição do relato verbal assumiu função de variável independente. Nesse estudo, estudantes universitários tinham, no elo do modelo, uma tarefa não verbal de escolha de acordo com o modelo. No elo do auto-relato, os participantes tinham que escolher alternativas que descreviam se eles tinham acertado ou não ("Sim" e "Não"). Na fase de linha de base (LB), os auto-relatos não produziam perda (nem ganho) de pontos. Na fase SIM, os auto-relatos "Sim" produziam a perda de 1 ponto. Na fase NÃO, os autorelatos "Não" produziam a perda de 1 ponto. $\mathrm{Na}$ primeira exposição à $\mathrm{LB}$, três dos quatro participantes apresentaram um viés para o auto-relato "Sim". Na fase SIM, todos os participantes diminuíram a freqüência de auto-relatos "Sim", enquanto na fase NÃO, a frequiência dos auto-relatos "Sim" aumentou. $\mathrm{Na}$ segunda exposição à $\mathrm{LB}$, três dos quatro participantes apresentaram um viés para o auto-relato da alternativa que não havia sido punida na fase imediatamente anterior. Esses resultados sugerem que uma história de punição afeta os auto-relatos, conforme sugerido por Critchfield (1993).

Comportamentos alternativos. Kramer (1982, Experimento 3) avaliou o efeito da emissão de comportamentos alternativos antes e após a apresentação do modelo, efeitos que são conhecidos na literatura como interferência pró-ativa e retroativa, respectivamente. No elo de modelo, pombos deveriam completar um esquema misto FR 1-FR 3 no disco central iluminado por uma luz vermelha e, em seguida, completar outro esquema misto FR 1-FR 3 no disco central iluminado por uma luz verde. Após completar o elo do modelo, era iniciado um intervalo que foi progressivamente manipulado (valores entre 0,1 e 4,0 s). Em seguida, no elo do auto-relato, dois discos laterais eram iluminados, em metade das tentativas, por uma luz vermelha e, na outra metade, por uma luz verde, devendo os animais relatar a razão de respostas emitida durante o modelo vermelho ou verde. Caso o esquema de menor razão houvesse sido completado, respostas no disco da esquerda produziam alimento, porém, se a maior razão é que houvesse sido emitida, respostas no disco da direita é que produziam reforços.

Uma vez que o modelo vermelho sempre ocorria antes do modelo verde, o efeito de comportamentos emitidos antes do modelo foi avaliado pela acurácia do auto-relato do modelo verde e o efeito de comportamentos emitidos após o modelo foi avaliado pela acurácia do auto-relato do modelo vermelho. Os resultados foram também analisados em termos das possíveis combinações de apresentação das alternativas no modelo: FR 1 (ou FR 3) no modelo vermelho seguido de FR 1 (ou FR 3) no modelo verde tentativas homogêneas; e FR 1 (ou FR 3) no modelo vermelho seguido por FR 3 (ou FR 1) no modelo verde - tentativas heterogêneas. Primeiramente, os resultados indicaram que comportamentos emitidos após o modelo afetaram mais substancialmente o auto-relato do que comportamentos emitidos antes do modelo, uma vez que a acurácia do auto-relato do modelo vermelho apresentou reduções maiores com o aumento do intervalo do que o auto-relato do modelo verde. Adicionalmente, o auto-relato de tentativas heterogêneas foi menos acurado do que o auto-relato de tentativas homogêneas, e aumentos no intervalo foram acompanhados por uma maior diminuição da acurácia do auto-relato de tentativas heterogêneas do que de tentativas homogêneas. Os autores concluíram que a emissão de outros comportamentos interfere no controle discriminativo exercido pelas contingências sobre o auto-relato.

Em conjunto, esses estudos mostraram que a acurácia do auto-relato é afetada por modificações nas contingências que estabelecem e mantêm esse comportamento. Aumentos na taxa/probabilidade/magnitude dos reforços para o relato de uma alternativa geram relatos superestimados da freqüência desses eventos, da mesma forma que uma história de punição de relatos de insucesso produzem relatos superestimados de sucesso. Além disso, os auto-relatos são também influenciados pela emissão de comportamentos antes ou após o comportamento a ser relatado.

\section{CONSIDERAÇÕES FINAIS}

As contingências de reforçamento alteram o comportamento, promovendo a organização e, conseqüentemente, a adaptação do comportamento ao ambiente. $\mathrm{O}$ comportamento adaptativo, nesse sentido, refere-se a um "conhecimento tácito" sobre as variáveis de controle do comportamento. Entretanto, um organismo pode apresentar um outro tipo de conhecimento sobre as contingências, um "conhecimento verbal", ou seja, as contingências que geram o comportamento adaptativo podem servir como estímulo discriminativo para um outro comportamento adaptativo, o de relatar (Shimp, 1983).

O auto-relato é um comportamento cotidiano relevante em diversos contextos. No contexto educacional, por exemplo, o aluno é solicitado a relatar suas idéias e críticas sobre o conteúdo 
ensinado. No contexto profissional, o empregado deve descrever as tarefas executadas e as dificuldades encontradas ao executá-las. Nas relações familiares, pais requerem que os filhos relatem suas experiências diárias, assim como os cônjuges esperam que seus parceiros compartilhem suas angústias e alegrias. Considerando-se que o acesso ao mundo privado dos indivíduos contribui para o estabelecimento de diversos tipos de relações interpessoais e que este acesso pode ser feito, mesmo que de forma parcial e/ou imprecisa, por meio do auto-relato, torna-se inestimável a compreensão das contingências responsáveis pela origem e manutenção desse comportamento. Na busca dessa compreensão, é importante considerar o que a pesquisa básica tem a dizer.

No caso específico do auto-relato, a pesquisa básica tem demonstrado que esse comportamento é afetado por características tanto do comportamento a ser relatado como das contingências que mantêm esse comportamento. Quais seriam as implicações desse achado para a vida cotidiana? De maneira geral, as pesquisas com sujeitos humanos e não humanos indicam que, $a$ despeito de $o$ referente (o comportamento e a contingência que o mantém) ser imutável, uma vez que já ocorreu, sua descrição pode variar, já que o comportamento de descrever pode estar sob o controle de diferentes aspectos do referente. A possibilidade de que múltiplos aspectos de uma contingência possam vir a exercer papel de estímulo discriminativo para o auto-relato assume um valor inestimável no contexto clínico, pois as verbalizações do cliente são o principal instrumento de trabalho do terapeuta, e um dos objetivos gerais da terapia, a despeito das queixas e demandas específicas de cada cliente, é promover autoconhecimento. Segundo Fester (1973), o autoconhecimento deve ser priorizado na terapia por dois motivos principais: (1) quando o indivíduo aprende a observar o ambiente, seu comportamento pode ficar sob o controle de aspectos que antes não assumiam funções discriminativas; e, (2) a descrição acurada do próprio comportamento e de suas variáveis de controle pode sugerir maneiras mais eficazes de alterar o ambiente. Assim sendo, é possível afirmar que o autoconhecimento seria um aspecto importante para o estabelecimento de autocontrole (Beckert, 2002).

Transpondo alguns dos achados da pesquisa básica para o contexto terapêutico, considere-se, por exemplo, que um cliente (Pedro) está relatando suas dificuldades em manter relações afetivas duradouras. Suponha que seus namoros mais significativos tenham sido com Marta e Lúcia. Marta era uma namorada muito ativa, gostava de sair muito para barzinhos, festas e boates; além disso, era alegre, extrovertida e muito carinhosa. Por outro lado, Lúcia era tranqüila, introspectiva e muito tímida. Ela e Pedro tinham uma vida pacata, preferindo ficar em casa assistindo filmes, escutando música, lendo livros. Ao analisar os motivos do término dessas relações, Pedro pode enfatizar diferentes aspectos do seu relacionamento com Marta e Lúcia, tais como a taxa de respostas ("eu saía muito com Marta, era exaustivo", "eu saía pouco com Lúcia, parecíamos um casal de idosos"), a densidade dos reforços que cada uma das namoradas apresentava para o seu comportamento ("Marta era excessivamente carinhosa, o que me sufocava"; "Lúcia era muito tímida, eu nunca tinha certeza se estava agradando ou não"), e assim por diante. Além disso, poderiam ocorrer vieses no auto-relato em decorrência do tempo transcorrido desde o término da relação e da possibilidade de terem ocorrido contingências competitivas. Isto é, se o relacionamento com Marta terminou recentemente, enquanto o relacionamento com Lúcia terminou 5 anos atrás, é bem provável que o auto-relato do namoro com Marta seja mais acurado; e se o cotidiano de Pedro fosse bastante conturbado por causa de problemas sérios no trabalho enquanto namorava Marta, mas fosse bastante tranquiilo enquanto namorava Lúcia, a correspondência entre o que foi relatado e o que realmente ocorreu seria mais alta neste último caso. Para o terapeuta é importante identificar por que o cliente está relatando tais aspectos das contingências, por que elas assumiram controle sobre seu comportamento para, então, discutir com ele formas alternativas de ação.

Outra contribuição da pesquisa básica refere-se à identificação das propriedades controladoras de fatores presentes nas contingências que estabelecem e mantêm o próprio auto-relato. Voltando ao exemplo anterior, a acurácia do auto-relato de Pedro também dependerá dos reforços contingentes a esse auto-relato. Aqui, há duas fontes de controle importantes: o reforçamento e punição presentes na história passada de Pedro e em sua relação atual com o terapeuta. Se Pedro tiver uma história caracterizada por punições de relatos de insucesso (e.g., acadêmicos, profissionais, afetivos), é bem provável que seu relato seja enviesado na direção de atribuir a Marta e a Lúcia a responsabilidade do término do namoro (a mentira, a negação e a omissão originam-se, freqüentemente, de punição). Adicionalmente, o terapeuta pode reforçar prematuramente determinados aspectos de auto-relato de Pedro, ou inadvertidamente punir outros aspectos, e 
isso pode prejudicar a discriminação das variáveis controladoras das dificuldades afetivas do cliente.

Em todas essas situações, com vista a promover auto-relatos acurados, ou alternativamente, a correspondência entre o fazer e o dizer, o terapeuta deve considerar que a freqüência, duração e complexidade do comportamento a ser descrito, e a freqüência e densidade dos reforços contingentes a esse comportamento, dentre outras variáveis, afetam o autorelato. Também não deve deixar de considerar sob que condições de reforçamento e punição o auto-relato foi estabelecido e é mantido. Esses aspectos são cruciais para o desenvolvimento de autoconhecimento e, conseqüentemente, para a ocorrência de autocontrole. Como apontado por Skinner (1974/2004), quanto mais acurado for o conhecimento que tivermos de nosso próprio comportamento (e do comportamento dos outros) e de suas contingências determinantes, maiores serão nossas chances de mudar o ambiente e, conseqüentemente, a nós mesmos.

\section{REFERÊNCIAS}

Beckert, M. E. (2002). Correspondência: quando o objetivo terapêutico é o "digo o que faço e faço o que digo". Em H. J. Guilhardi, M. B. B. P. Madi, P. P. Queiroz \& M. C. Scoz (Orgs.), Sobre comportamento e cognição: contribuições para a construção da teoria do comportamento (pp.183-194). Santo André: ESETec.

Beckert, M. E. (2005). Correspondência verbal/não-verbal: pesquisa básica e aplicações na clínica. Em J. AbreuRodrigues \& M. R. Ribeiro (Orgs.), Análise do comportamento: pesquisa, teoria e aplicação (pp. 229-244). Porto Alegre: Artmed.

Commons, M. L. (1979). Decision rules and signal detectability in a reinforcement-density discrimination. Journal of the Experimental Analysis of Behavior, 32, 101-120.

Critchfield, T. S. (1993). Signal-detection properties of verbal selfreports. Journal of the Experimental Analysis of Behavior, 60, 495-514.

Dymond, S. \& Barnes, D. (1997). Behavior-analytic approaches to self-awareness. The Psychological Record, 47, 181-200.

Fester, C. B. (1973). A functional analysis of depression. American Psychologist, 28, 857-870.

Hobson, S. L. (1975). Discriminability of fixed-ratio schedules for pigeons: Effects of absolute ratio size. Journal of the Experimental Analysis of Behavior, 23, 25-35.

Jones, B. M. \& Davison, M. (1998). Reporting contingencies of reinforcement in concurrent schedules. Journal of the Experimental Analysis of Behavior, 69, 161-183.

Killeen, P. R. (1978). Superstition: A matter of bias, not detectability. Science, 199, 88-90.
Kramer, S. P. (1982). Memory for recent behavior in the pigeon. Journal of the Experimental Analysis of Behavior, 38, 71-85.

Lattal, K. A. (1975). Reinforcement contingencies as discriminative stimuli. Journal of the Experimental Analysis of Behavior, 23, 241-246.

Lattal, K. A. (1979). Reinforcement contingencies as discriminative stimuli: II - Effects of changes in stimulus probability. Journal of the Experimental Analysis of Behavior, $31,15-22$.

Lattal, K. A. \& Doepke, K. J. (2001). Correspondence as conditional stimulus control: Insights from experiments with pigeons. Journal of the Experimental Analysis of Bebavior, 34, 127-144.

Lionello-DeNolf, K. M., \& Urcuioli, P. J. (2003). A procedure for generating differential "sample" responding without different extereoceptive stimuli. Journal of the Experimental Analysis of Behavior, 79, 21-35.

Maki, W. S. (1979). Discrimination learning without short-term memory: Dissociation of memory processes in pigeons. Science, 204, 83-85.

Okouchi, H. (2003). Effects of differences in interreinforcer intervals between past and current schedules on fixed-interval responding. Journal of the Experimental Analysis of Behavior, 79, $49-64$.

Okouchi, H. \& Songmi, K. (2004). Differential reinforcement of human self-reports about schedule performances. The Psychological Record, 54, 461-478.

Pliskoff, S. S. \& Goldiamond, I. (1966). Some discriminative properties of fixed-ratio performance in the pigeon. Journal of the Experimental Analysis of Behavior, 9, 1-9.

Reynolds, G. S. (1966). Discrimination and emission of temporal intervals by pigeons. Journal of the Experimental Analysis of Behavior, 9, 65-68.

Reynolds, G. S. \& Catania, A. C. (1962). Temporal discrimination in pigeons. Science, 135, 314-315.

Rilling, M. \& McDiarmid, C. (1965). Signal detection in fixedratio schedules. Science, 148, 526-527.

Sanabio, E. T. \& Abreu-Rodrigues, J. (2002). Efeitos de contingências de punição sobre o comportamento verbal e nãoverbal. Psicologia: Teoria e Pesquisa, 18, 161-172.

Shimp, C. P. (1981). The local organization of behavior: Discrimination of and memory for simple behavioral patterns. Journal of the Experimental Analysis of Behavior, 36, 3033015.

Shimp, C. P. (1982). On metaknowledge in the pigeon: An organism's knowledge about its own behavior. Animal Learning \& Behavior, 10, 358-364.

Shimp, C. P. (1983). The local organization of behavior: Dissociations between a pigeon's behavior and self-reports of that behavior. Journal of the Experimental Analysis of Behavior, 39, 61-68.

Skinner, B. F. (2000). Ciência e comportamento humano. São Paulo: Martins Fontes. (Original publicado em 1953).

Skinner, B. F. (2004). Sobre o Behaviorismo. São Paulo: Cultrix. (Original publicado em 1974). 
Souza, A. S. (2006). Discriminação de contingências de variação $e$ repetição. Dissertação de Mestrado Não-Publicada, Programa de Pós-Graduação em Psicologia, Universidade de Brasília, Brasília.

Urcuioli, P. J. (1985). On the role of differential sample behavior in matching-to-sample. Journal of Experimental Psychology: Animal Behavior Processes, 11, 502-519.

Urcuioli, P. J. \& DeMarse, T. (1994). On the relationship between differential outcomes and differential sample responding in matching-to-sample. Journal of Experimental Psychology: Animal Behavior Processes, 20, 249-263.
Ziriax, J. M. \& Silberberg, A. (1978). Discrimination and emission of different key-peck durations in the pigeon. Journal of Experimental Psychology: Animal Behavior Processes, 4, 121.

Recebido em 09/02/2006

Aceito em 20/09/2006

Endereço para correspondência: Josele Abreu-Rodrigues. Universidade de Brasília, Instituto de Psicologia, CEP 79.910-900, Brasília-DF. E-mail: abreu@unb.br 